\title{
Research on Global Positioning System in Mobile Communication Equipment Based on Android Platform
}

\author{
Huang Yi, He Qian, Wang Yubin, Xie Zhiyong, Wang tao \\ Nanchang Business College in Jiangxi Agricultural University, NO.888, Mount Lu Avenue 330044
}

\begin{abstract}
Global Positioning System in Android mobile communication equipment has been widely applied for its good performance in smart phone. Hence, to improve the precision of GPS navigation in Android platform attaches great importance to the development of modern high-tech products. The paper presents a new method on how to enhance the precision of GPS positioning by processing the original data of GPS satellite, then analyzing the data and count the possible error from the connection of the data from satellites.
\end{abstract}

Index Terms - Android, Global Positioning System

\section{Introduction of Android}

Android is a Linux-based operating system designed primarily for touchscreen mobile devices such as smart phones and tablet computers. Android was unveiled in 2007 along with the founding of the Open Handset Alliance Android is open source and Google releases the code under the Apache License.

The framework of Android system can be classified into 3 layers---Operation System, Application and Middleware. The core of Operation System is Linux-based framework which provides the basic structure for Android system; while the Application is formed by application programs. This is the managing platform for services of Android system, and it is and vital part of the entire framework. The third layer is composed of function library and Android run time. Component of functions like image, media, SSL \& webkit and SSL \& webkit combine to function on this layer. The fourth layer is the application of Android offering services for Android application.

Dalvik is the process virtual machine (VM) in Google's Android operating system. Dalvik is thus an integral part of Android, They are then converted from Java Virtual Machine-compatible class files to Dalvik-compatible .dex (Dalvik Executable) files before installation on a device. Dalvik is open-source software. Every Dalvik application can be exerted as a independent Linux process, so the process is stable in operation.

Android system is typically used on mobile devices such as mobile phones and tablet computers Programs are commonly written in Java and compiled to bytecode. Dalvik virtual machine has been improved and optimized to be applied on mini-scale platform like smart phones, for example, to decrease the usage of RAM by using processing unit, the increase the efficiency of processor, to execute plurality of programs simultaneously.

Android provide a majority of standard function library for software. DalvikVM is needed to identify the source code of software because those functions to some extent are encrypted. Plus, there are many Android exclusive functions for the user to dial and position easily.

Within Android system, function library and dalvik Virtual Machine form the Android run time, the middle part. Its features are:

- Java program should be transferred by DalvikVM into DEX to execute

- Functions must be defined and claimed in main function before take into execution.

- Improper form of function and lack of Android-exclusive function to execute function will result in the failure of Android application in Java Runtime.

\section{Android GPS Positioning Technology}

A. GPS System

The Global Positioning System (GPS) is a space-based satellite navigation system that provides location and time information in all weather conditions, anywhere on or near the Earth where there is an unobstructed line of sight to four or more GPS satellites. Satellites, ground control system and receivers comprise GPS system. There are 24 artificial satellites, 3 of them are back-up. All these satellites spread evenly on the orbit centered on earth. Every satellite sends out radio signals of 2 ratio returning earth for the receivers to locate; ground control system includes a primary control station and four monitoring stations. The main task of ground control system is to monitor the status of satellite on operation, and to analyze the deviations and changes of data to compute the error and send the rectified data to satellite. The receivers locate the wires via catchers.

\section{B. GPS positioning technology}

To build net connection is the first step to realize GPS positioning. Mobile communication devices $\log$ on net by GPRS or Wi-fi(a popular technology that allows an electronic device to exchange data wirelessly (using radio waves) over a computer network, including high-speed Internet connections.), then build connection with system background server by SOCKET(an end-point in a communication across a network or the Internet). The built-in GPS navigation module search for positioning data from satellites, then analyze the locator data and status information from the longitude, latitude and directions. Next, the resultant data are uploaded to Background monitoring server for processing so as to locate and monitor and user cell phones. 


\section{The principles of GPS navigation in Android-based Smart phone}

The positioning in Android-based smart phone is completed by the built-in locator module, involving navigation software, GSM communication module and GPS chip. They receive positioning signals from satellite continuously and GPS module figure out the related information through analysis, like direction, or speed of target.

The following three modules are musts for GPS navigation in Android-based smart phone.

1) GPS module is the essence in functioning; it collects data of tracking and positioning from satellites.

2) Map Navigation software helps to visualize the data from GPS module. The software refreshes the e-map with the data to make sure the positioning spontaneously.

3) GSM communication module analyzes the data from the other two module and upload the result to the designed website.

\section{Critical code for GPS Positioning Navigation}

Through software, the call of GPS positioning navigation critical code can be realized, the following code are listed by Create View in simple txt format.

Public void on Create View ()

$\{$ GpsView = new Gps View (this); $\}$

This method calls the formation of object in GPS view, it can be presented in a similar way of GPS view

Public class Gps View extends View

\{My Locate ovedord;

Public Gps View (Loeate Me pCtx)

\{ super (pCtx);

Ovedolord =pCtx; $\}$

public void on Draw(Canvas canvs)

$\{$ Paintp $=$ new Paint () ;

String Strlat="Latitude:"+ over lord. Get Lat ();

String StrLon="longitude: "+ over lord. getL On ();

Canvs. Draw Text (StrLat,32,32,p);

Canvs. Draw Text (StrLon,32,44,p);\}\}

The above procedure code of GPS View is capable of analyzing the test data of longitude and latitude, and showing them on screen via on Draw. On Draw calls Super class formation to reach Interruption Command of Activity Pointer and locates by analyzing the connected data.

\section{GPS Positioning Data Analysis in Mobile Communication Equipment}

The relationship of hardware and software of GPS Positioning system on Android-based smartphone is illustrated in fig.3. When the program is started, it sends positioning signal to satellite. Satellite returns corresponding data to cellphone for its present location information.

\section{A. Judgment of abnormal data}

The data from GPS positioning system fail to be absolute precise for its precision will be affected by climate or other factors. In this case, the data deviated from those obtained in ordinary situations are called abnormal data. In order to the decrease error, Multi-point diagnosis is needed to analyze abnormal data for times. Generally, statistical method, deviation method and Density method are mainly adopted to deal with the abnormal data.

Time series method is a universal method to analyze the error in Android -based smart phone GPS positioning, which predicts the data in accordance with the time series.

In the paper, serial variance is a standard to estimate whether is abnormal or not. When the serial variance exceeds $3 \sigma$ (the range within the data changes for GPS positioning), the test value should be scattered nearby the actual value $\mathrm{x}_{0}$, the average value the normal estimate for $\mathrm{x}_{0}$ otherwise data are conceived as abnormal. Average value and variance can be counted in recurrence formula

$$
\begin{gathered}
\bar{x}_{n}=\bar{x}_{n-1}+\frac{x_{n}-\bar{x}_{n-1}}{n} \\
\sigma_{n}^{2}=\frac{n-2}{n-1} \sigma_{n-1}^{2}+\frac{\left(x_{n}-\bar{x}_{n-1}\right)^{2}}{n}
\end{gathered}
$$

$\bar{x}_{n}$ represents the average value, $\sigma_{n}^{2}$ is variance, the test valued received is $x_{n}$. From $\left|x_{n}-\bar{x}_{n}\right|>3 \sigma^{2}$, the new average value and variance are obtained. If $x_{n}$ is beyond desired range, it should be deleted. Then repeat the method until all the abnormal data are eliminated. The connected of data will be further analyzed ensuring the entireness of data and minimize the error.

\section{B. The Analysis the Positioning Data.}

The accuracy of the GPS positioning depends chiefly on two factors-----the quantity of the GPS satellite responsible for position combination and the distribution of satellites. Because these factors shows the accuracy GDOP value (Geometric dilution of precision, a major standard to judge the accuracy for a positioning system). The connection between the amount of satellites and the influence of positioning will be discussed in the following part.

Table1 the statistics of GDOP, PDOP average values

\begin{tabular}{|l|c|c|c|c|}
\hline Cut-off point & 10 & 20 & 30 & 40 \\
\hline GDOP & 1028 & 2.92 & 3.99 & 8.36 \\
\cline { 1 - 4 } GPS & 1.74 & 2.35 & 4.02 & 7.30 \\
\hline PDOP ((0.5--99.9)) & & & & \\
\hline GPS &
\end{tabular}

Table 1 shows that the inner height angle lowers than $20^{\circ}$ in a day with the movement of earth and there are at most four satellites to function, GPS can function smoothly in this circumstance. In other words, data retrieved in this case exist conspicuous error and should be analyzed.

The development of GPS positioning in Android-based smart phone demands clean and pollution-free observation data. Through process analysis, the error is strikingly lessened 
and the accuracy improved correspondingly as long as the parameters of prototype conform to the basic requirements. In general, simulation result proves the practicability of solution application of GPS positioning which can enhance the precision of GPS positioning and reduce the error. Data missing is the great obstacle for accurate positioning in smart phone. However, this problem will be solved by analysis data from satellite so as to increase the precision of GPS positioning remarkably.

\section{Conclusion and Prospect}

The GPS positioning technology in Android-based smart phone becomes advanced with the mutual effort of Google and its alliance member. The software for this technology is various. Since the official operation of GPS, its wide application brings conveniences in the military and civilian fields and facilitates people's life. However, the gap between the technology in China and the developed countries is notable. To research and develop GPS positioning system in smart phone attaches significance to the progress of China's technology, above more, it will revolutionize people's life and work.

With the enhancement and improvement of GPS, the positioning technology betters everyday. So does the GPS satellites navigation and the function of multitude and electronic gadgets. Based on the wide application of GPS positioning technology in Android-based smart phone, we set off the research on this field, wishing to provide cell phone users better service.

The paper studies the application and development of GPS positioning system on Android based cellphone, using the data retrieved from satellite. All-weather, global mode coverage, and high-precision enables the GPS to be employed to position in various situations. The location information is showed dynamically in Google Map on smart phone. This will be the future trend of the technology development.

\section{References}

[1] Chen Haida. "Development on GPS Positioning Track Dynamic Tester", February 2004.

[2] Ding Penghui. "Research and Realization of GPS Non-difference Phase Precise Positioning Algorithm", June 2008.

[3] Zeng Xinyi. "Design and Implementation of GPS Navigation System based on Android Platform", Computer and Modernization, vol. 9, 2012.

[4] Cai Libing. "Design of Implementation of GPS Positioning System based on Android Smart Mobile Devices", Computer Knowledge and Technology, December 2012.

[5] Zhou Feng. "Development of GPS based on Android Smart Phone Platform", November 2011. 